\title{
LA-UR-16-22317
}

Approved for public release; distribution is unlimited.

Title: $\quad$ LANL's Weather Machine and Comparison of KLAM with Nearby Wind Data

Author(s): $\quad$ Bruggeman, David Alan

Intended for: Presentation

Issued: 
Disclaimer:

Los Alamos National Laboratory, an affirmative action/equal opportunity employer,is operated by the Los Alamos National Security, LLC for the National NuclearSecurity Administration of the U.S. Department of Energy under contract DE-AC52-06NA25396. By approving this article, the publisher recognizes that the U.S. Government retains nonexclusive, royalty-free license to publish or reproduce the published form of this contribution, or to allow others to do so, for U.S. Government purposes. Los Alamos National Laboratory requests that the publisher identify this article as work performed under the auspices of the U.S. Departmentof Energy. Los Alamos National Laboratory strongly supports academic freedom and a researcher's right to publish; as an institution, however, the Laboratory does not endorse the viewpoint of a publication or guarantee its technical correctness. 


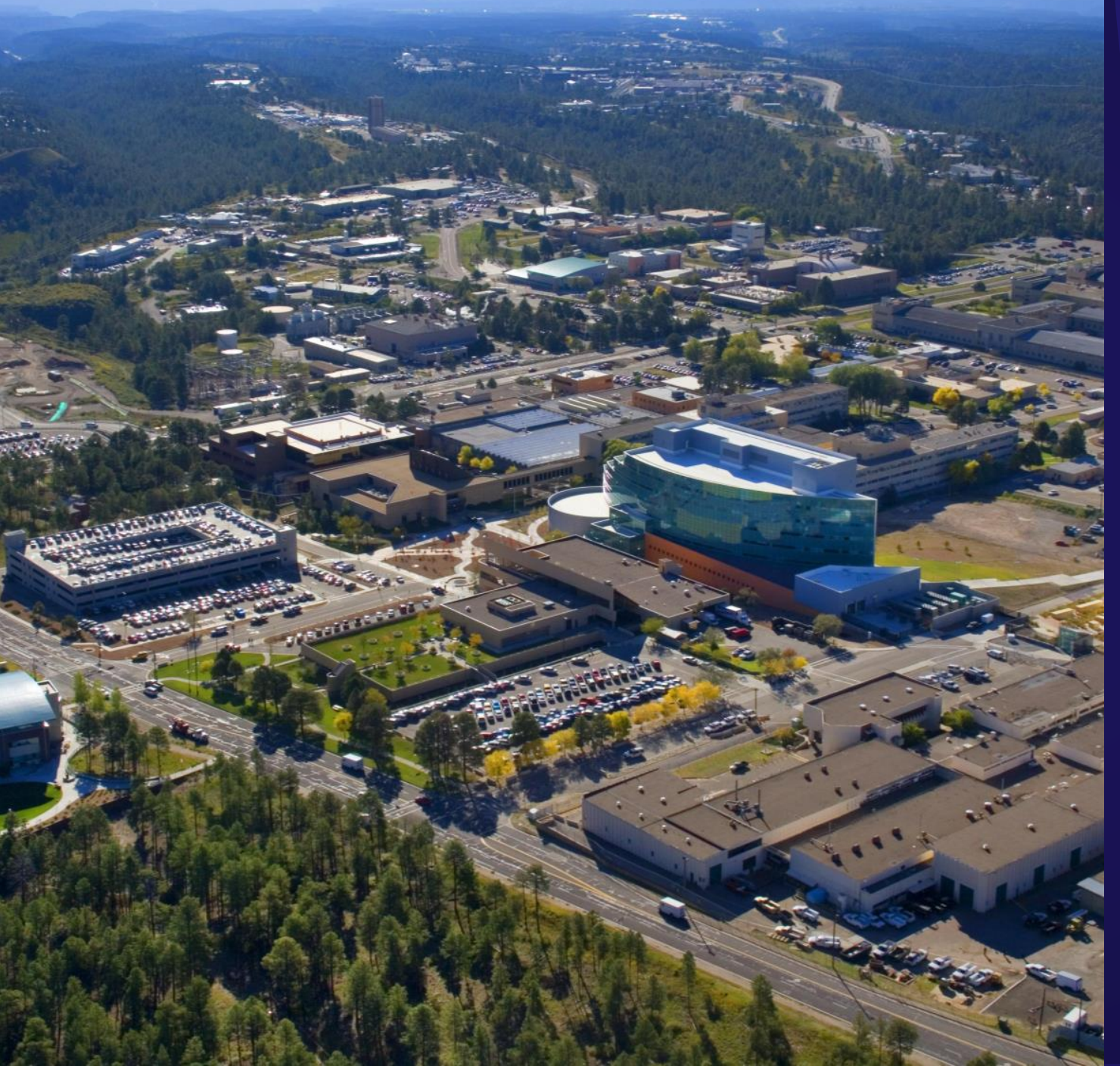

$\overbrace{-L o s}$ Alamos NATIONAL LABORATORY

EST. 1943

Delivering science and technology

to protect our nation and promote world stability 


\section{LANL's Weather Machine and Comparison of KLAM with Nearby Wind Data}

David Bruggeman

April 172016 


\section{Weather Monitoring}

- Meteorological monitoring in Los Alamos since 1910

- Five meteorology towers and a SODAR across LANL

- Data can be accessed on the Weather Machine

- http://environweb.lanl.gov/weathermachine

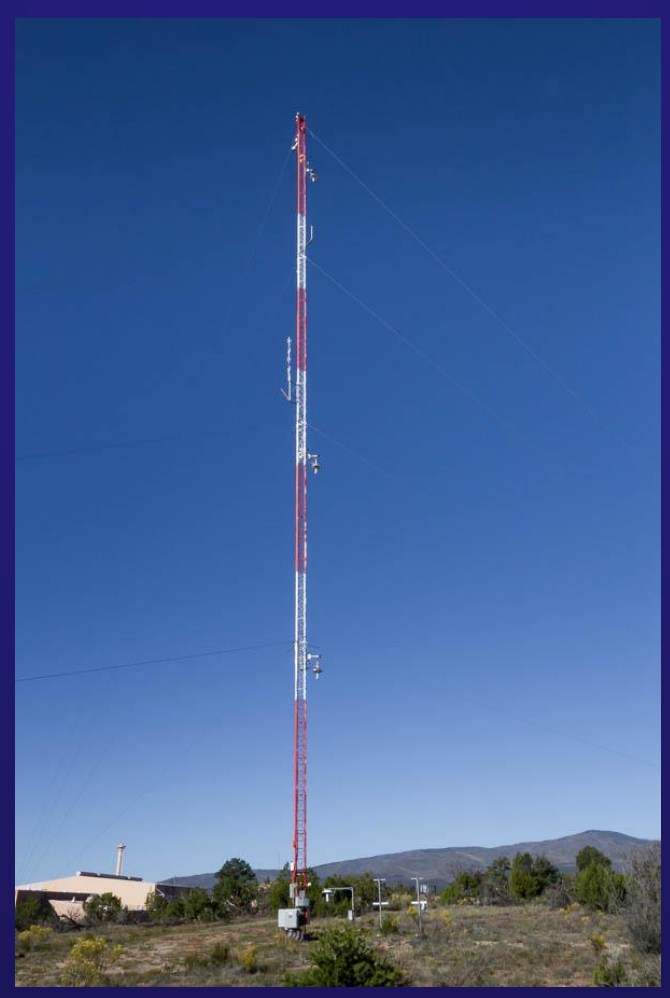




\section{Meteorological Monitoring Sites}

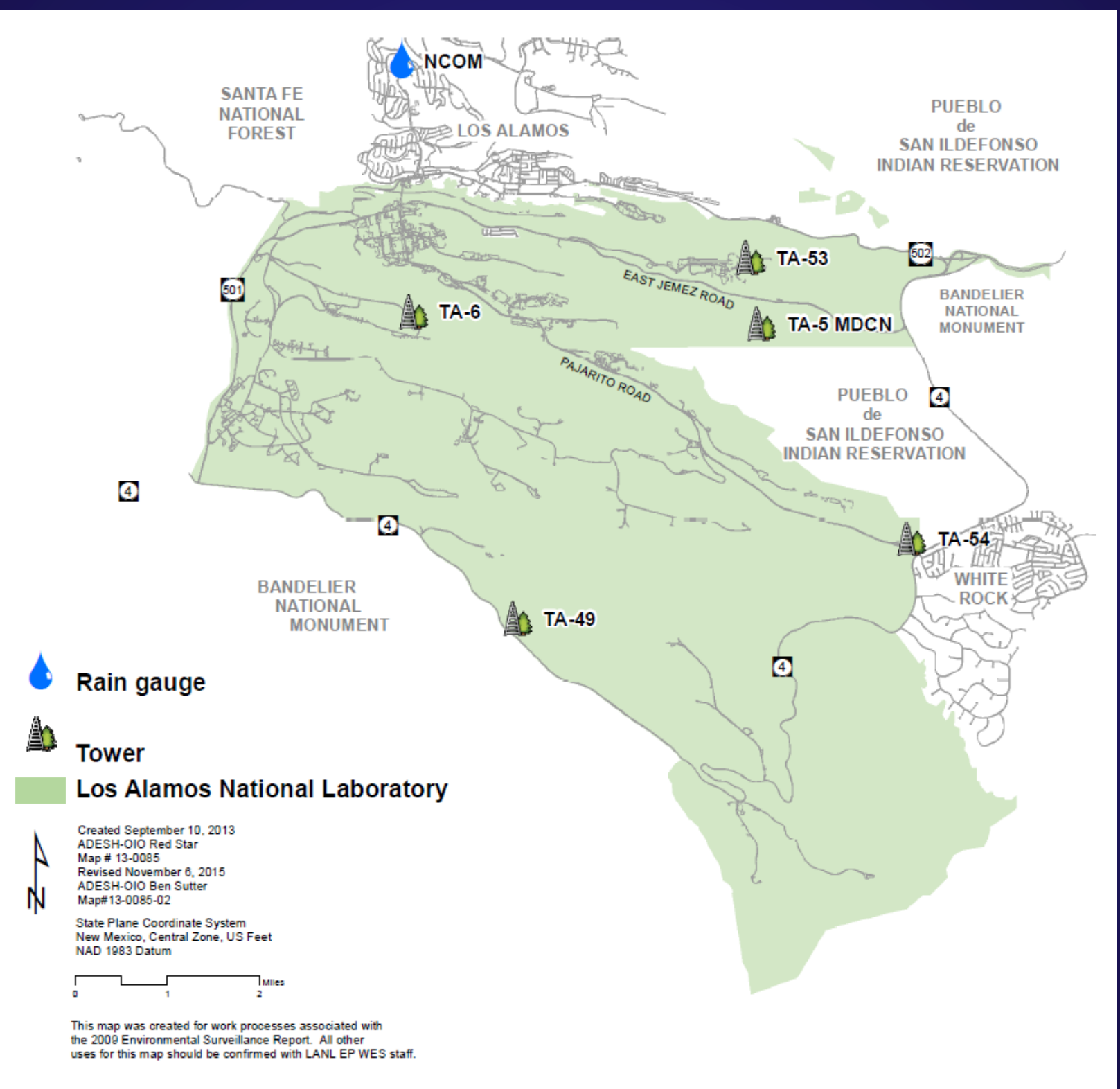




\section{Weather Machine}

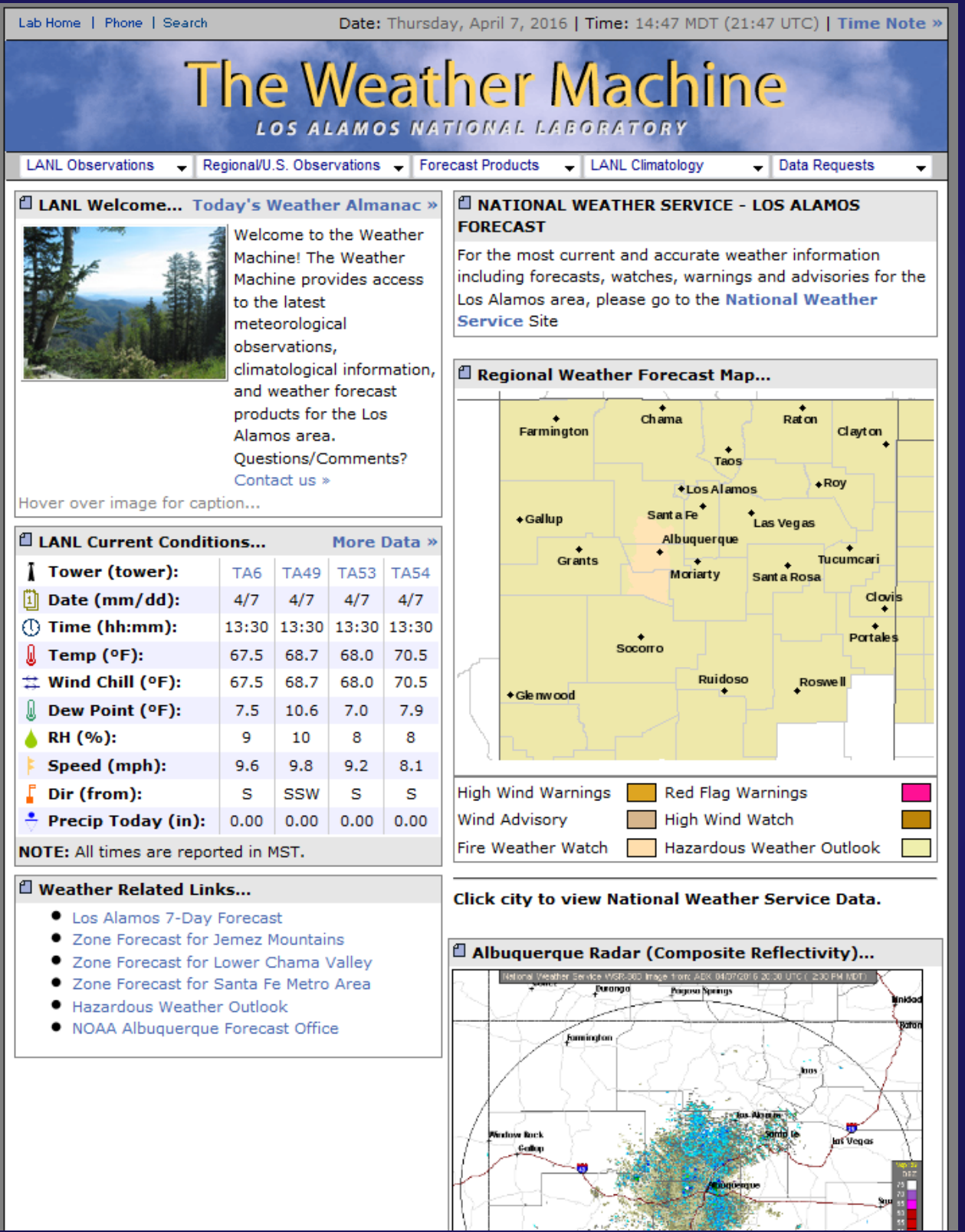




\section{Weather Machine - Wind Vector Field}

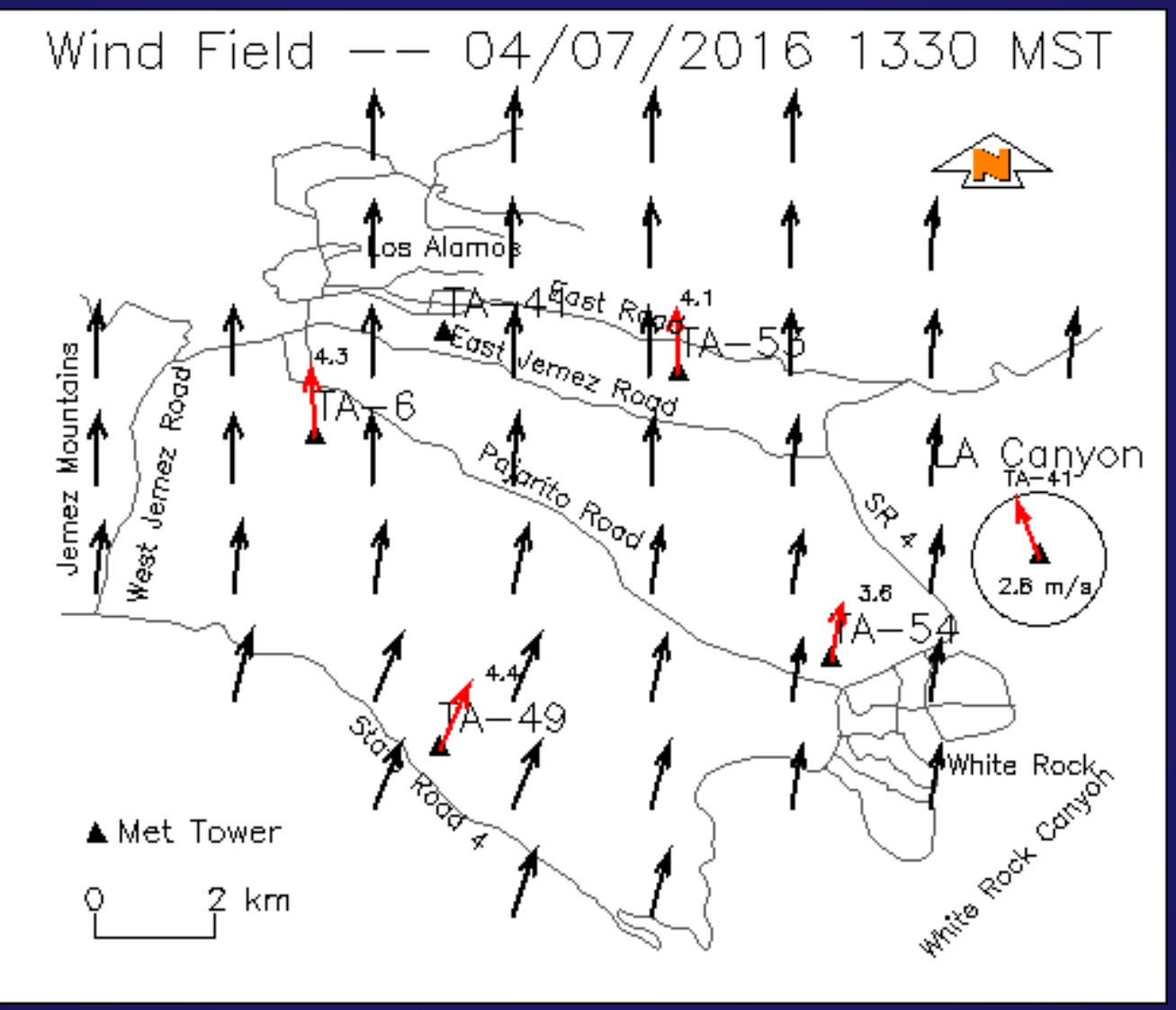




\section{Weather Machine - Raw Data Request}

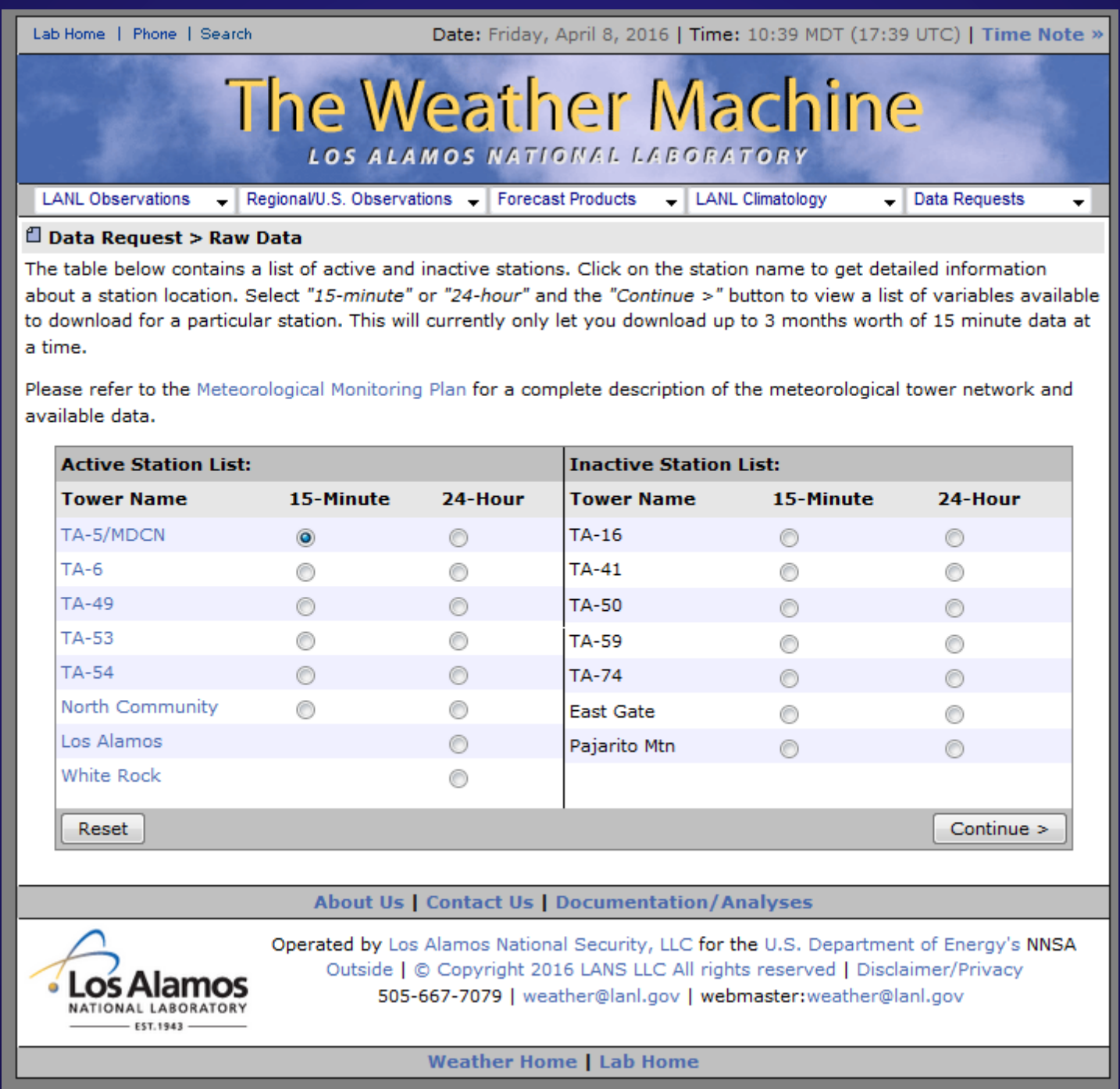




\section{Weather Machine - SODAR}

- Uses sound waves to measure wind and temperature up to $\sim 1 \mathrm{~km}$
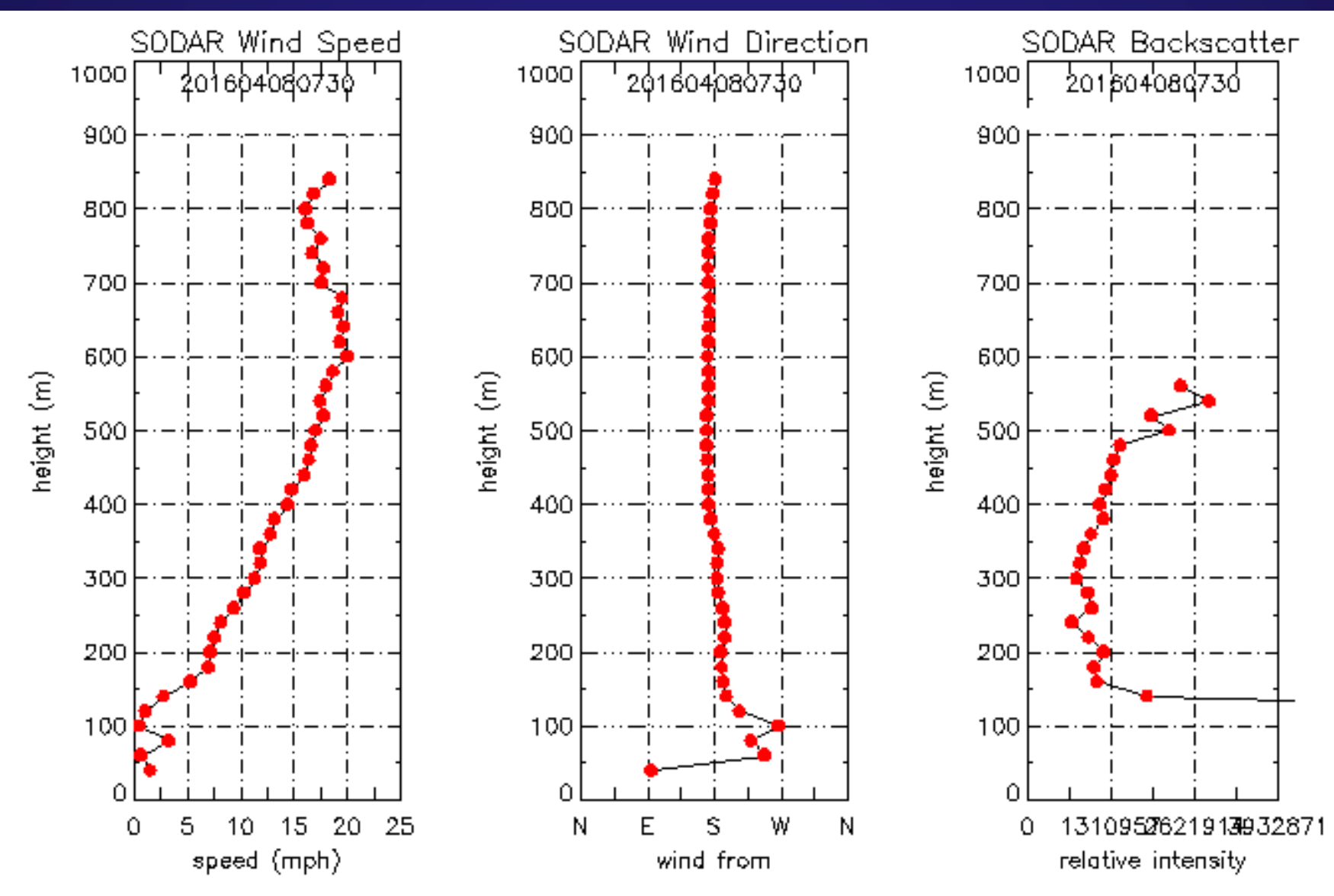


\section{Weather Machine - SODAR}

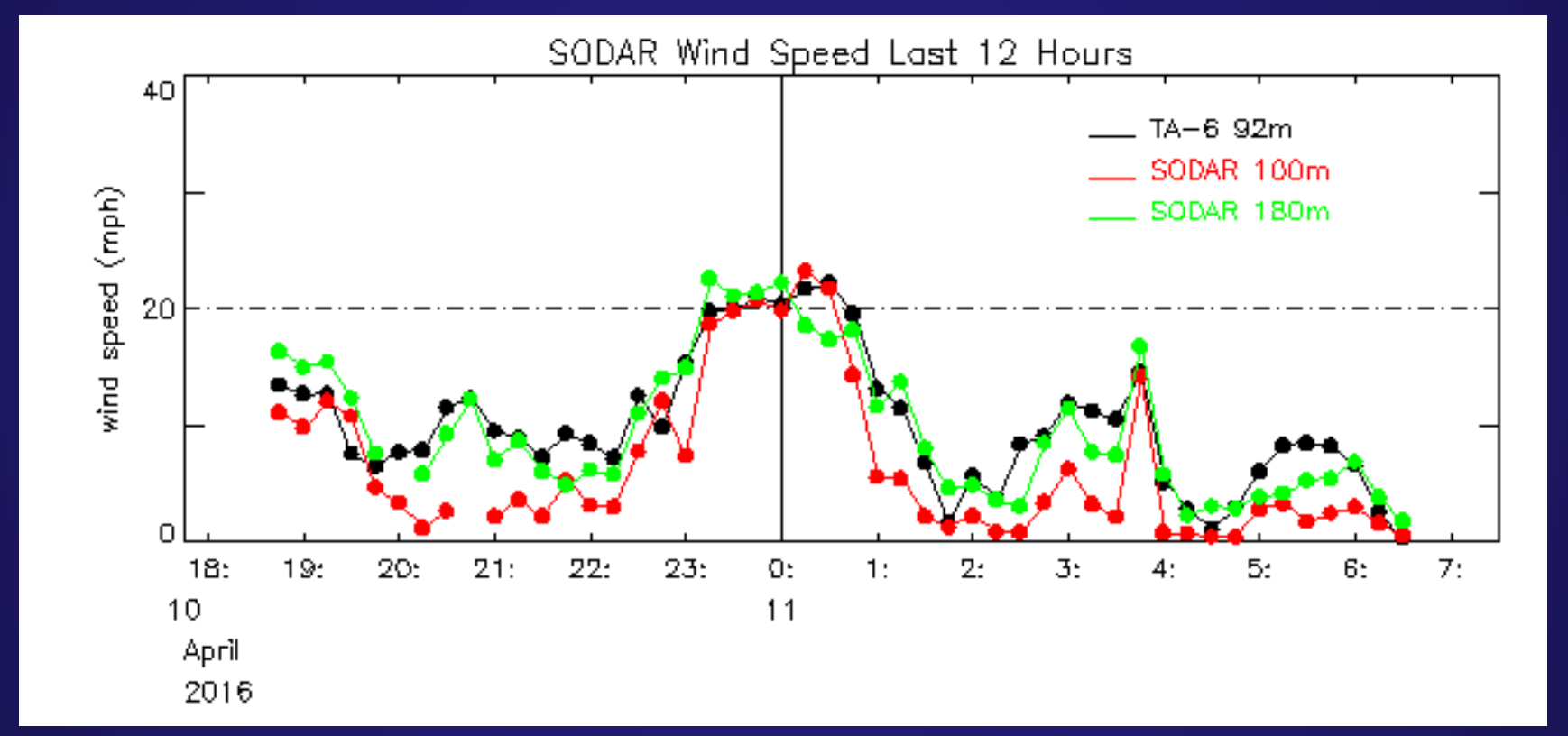




\section{Comparison of KLAM and TA-53}




\section{TA-53 \& KLAM Locations}

- TA-53 4,000 ft (1.2 km) away from KLAM

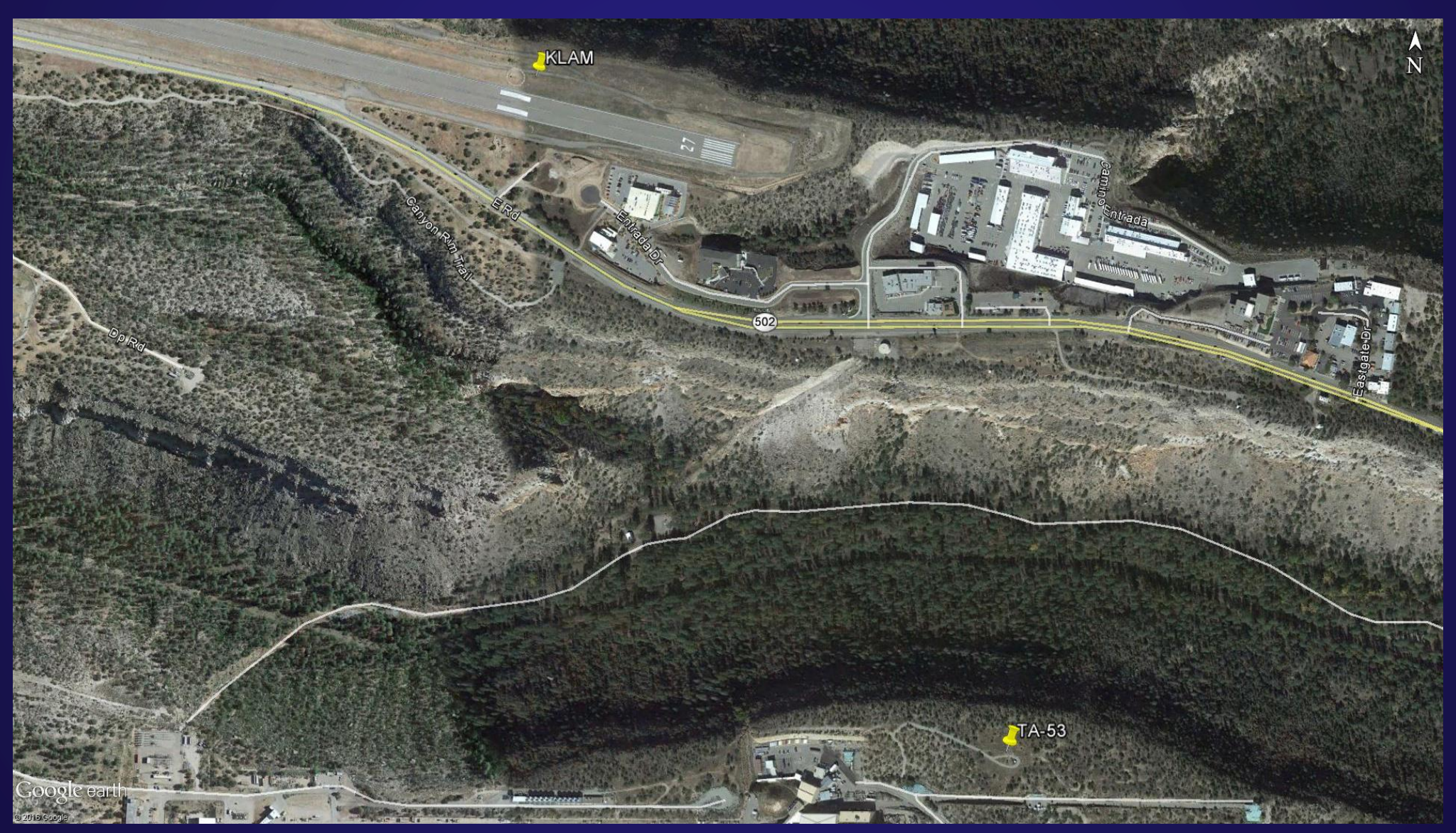




\section{Differences Between TA-53 and KLAM}

\begin{tabular}{|c|c|c|}
\hline Variables & TA-53 & KLAM \\
\hline Temperature near surface & $\checkmark$ & $\checkmark$ \\
\hline Temperature at upper levels & $\checkmark$ & X \\
\hline High/low temperatures & $\checkmark$ & $\checkmark$ \\
\hline Dew point & $\checkmark$ & $\checkmark$ \\
\hline Relative humidity & $\checkmark$ & $\checkmark$ \\
\hline Wind speed/direction & $\checkmark$ & $\checkmark$ \\
\hline Vertical wind speed & $\checkmark$ & X \\
\hline Wind gust & $\checkmark$ & $\checkmark$ \\
\hline Wind data at upper levels & $\checkmark$ & X \\
\hline Cloud layer heights & X & $\checkmark$ \\
\hline Precipitation 1,3,6,24 hours & $\checkmark \mathbf{X}$ & $\checkmark$ \\
\hline Visibility & X & $\checkmark$ \\
\hline Incoming shortwave radiation & $\checkmark$ & X \\
\hline
\end{tabular}




\section{Differences Between TA-53 and KLAM}

\begin{tabular}{|c|c|c|}
\hline & TA-53 & KLAM \\
\hline Anemometer & Propeller & 3-cup \\
\hline Elevation & $2130 \mathrm{~m}$ & $2186 \mathrm{~m}$ \\
\hline Anemometer height & $11.5 \mathrm{~m}$ (+2 upper levels) & $10 \mathrm{~m}$ \\
\hline Sampling period & $15-\min (0,15,30,45)$ & $20-\min (15,35,55)$ \\
\hline Precision & $\begin{array}{c}0.1 \mathrm{~m} / \mathrm{s} \\
<0.4 \mathrm{~m} / \mathrm{s}=0 \mathrm{~m} / \mathrm{s}\end{array}$ & $\begin{array}{c}\sim 0.5 \mathrm{~m} / \mathrm{s} \\
(1.5,2.1,2.6,3.1 \text { etc } \ldots) \\
<1.5 \mathrm{~m} / \mathrm{s}=0 \mathrm{~m} / \mathrm{s}\end{array}$ \\
\hline
\end{tabular}



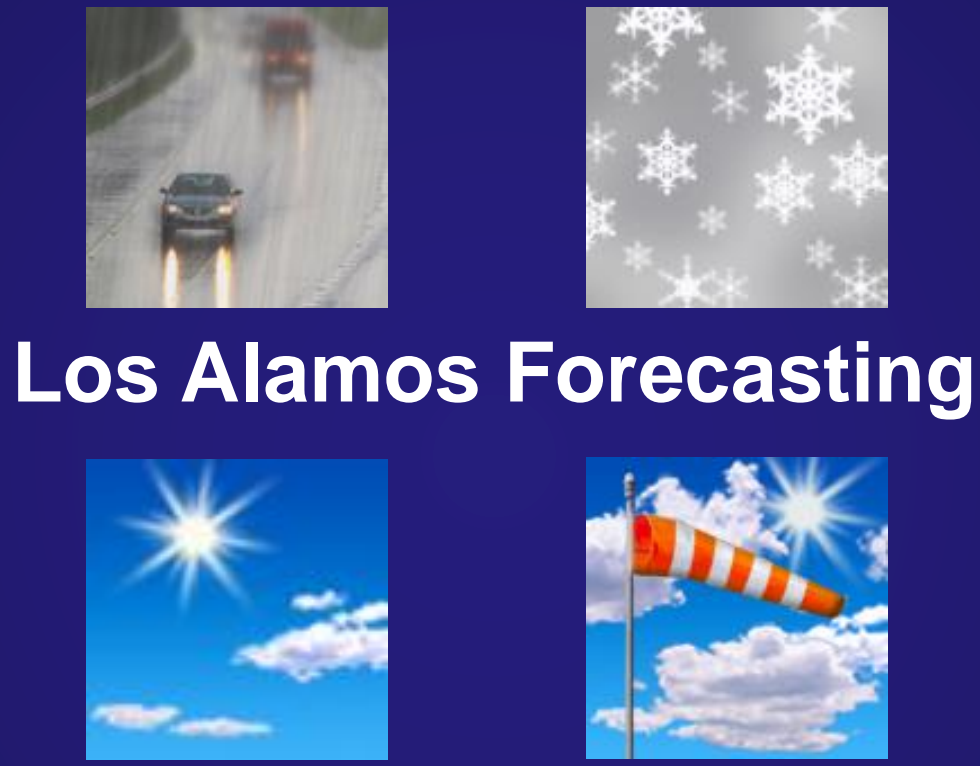